\title{
Tuning the tunnel coupling of quantum dot molecules with longitudinal magnetic fields
}

\author{
J. I. Climente ${ }^{a}$ \\ Departament de Química Física i Analítica, Universitat Jaume I, Box 224, E-12080 Castelló, Spain
}

(Received 23 October 2008; accepted 11 November 2008; published online 3 December 2008)

\begin{abstract}
We show that the energy splitting between the bonding and antibonding molecular states of holes in vertically stacked quantum dots can be tuned using longitudinal magnetic fields. With increasing field, the energy splitting first decreases to zero and then to negative values, which implies a bonding-to-antibonding ground state transition. This effect is a consequence of the enhancement of the valence band spin-orbit interaction induced by the magnetic field; it provides a flexible mechanism to switch the molecular ground state from bonding to antibonding. (C) 2008 American Institute of Physics. [DOI: 10.1063/1.3040058]
\end{abstract}

When two semiconductor quantum dots (QDs) are placed close to each other, the atomiclike states of the individual dots hybridize, forming bonding (nodeless) and antibonding (noded) molecularlike states, in analogy with diatomic molecules. ${ }^{1-4}$ The energy splitting between the bonding and antibonding states is given by tunnel coupling strength, i.e., the overlap between the atomiclike orbitals in the interdot barrier. The ability to manipulate this coupling in a controllable way while preserving the quantum coherence is important for the development of various device applications of coupled QDs (CQDs) in spintronics, ${ }^{5}$ optoelectronics, ${ }^{6}$ photovoltaics, ${ }^{7}$ and quantum information technologies. ${ }^{4,8,9}$

In electrostatically confined CQDs, where dots are usually laterally coupled, accurate control can be achieved through the gate voltage or perpendicular magnetic fields. ${ }^{10}$ In vertically CQDs however the degree of control achieved to date is comparatively lower. In these structures, the potential barrier height is fixed by the band offset between the QD and the surrounding matrix materials. By increasing the barrier length, one can reduce the tunnel coupling and hence the splitting between bonding and antibonding levels $\Delta_{\mathrm{BAB}},{ }^{9,11,12}$ but this can only be done during the sample growth. A more flexible method is the use of transverse magnetic fields that enable one to tune the tunnel coupling of a sample after its growth. ${ }^{13-15}$ Yet, large transverse fields may be required to obtain a sizable reduction in $\Delta_{\mathrm{BAB}}$ because the vertical confinement of these structures is usually strong and the axial symmetry of the structure is broken by the field. The latter effect activates otherwise forbidden transitions that are undesirable for optical manipulation and nondestructive measurements. ${ }^{16}$ The use of longitudinal magnetic fields would clearly be more desirable, but it has been shown that they barely affect the molecular coupling of electrons except in some particular setups, such as asymmetric coupled quantum rings. 17

In this work we show that an unprecedented degree of control on the tunnel coupling of vertically CQDs can be achieved with longitudinal magnetic fields if instead of using conduction electrons, one uses valence holes. The method can be applied to regular self-assembled or lithographically grown QDs and is particularly suitable for quantum information devices, where holes may outperform electrons due to

${ }^{a)}$ Electronic mail: climente@qfa.uji.es. their reduced spin relaxation and decoherence rates. ${ }^{18}$ The method follows from recent theoretical ${ }^{19}$ and experimental ${ }^{20}$ findings, which revealed that the valence band mixing of holes was responsible for a striking bonding-to-antibonding reversal of the ground state of CQDs with increasing interdot distance. Here we show that such a reversal can be also induced by a longitudinal magnetic field as it enhances the valence band mixing.

In order to investigate this effect we describe the hole states in CQDs using a four-band Luttinger-Kohn $k p$ Hamiltonian, which includes heavy hole $(\mathrm{HH})$ and light hole $(\mathrm{LH})$ coupling via spin-orbit (SO) interaction. Details about the theoretical method are given in Ref. 19. This model correctly described the qualitative features observed in related experiments. ${ }^{20} \mathrm{~A}$ magnetic field along the $z$ direction is included using a vector potential in the symmetric gauge, $\mathbf{A}$ $=B / 2(-y, x, 0){ }^{21}$ The spin Zeeman splitting is neglected as it simply provides a small numerical correction to the orbital effects discussed here. The QDs we consider are disk shaped and they have circular symmetry. Thus, the CQD potential reads $V(\rho, z)=V(\rho)+V(z)$. Here, $V(\rho)$ is an infinite well and $V(z)$ is a double rectangular well, whose value is zero inside the dots, $V_{c}$ (the band-offset potential) in the interdot region and infinite elsewhere. The lowest hole states are the Luttinger spinors with total angular momentum (Bloch +envelope) $z$-component $F_{z}=+3 / 2$, and chirality symmetry up $(\nu=\uparrow)$ or down $(\nu=\downarrow),{ }^{19}$

$$
\left|F_{z}=3 / 2, \nu=\uparrow\right\rangle=\left(\begin{array}{c}
c_{+3 / 2} f_{0}(\rho, \theta) \xi_{b}(z)\left|J_{z}=+\frac{3}{2}\right\rangle \\
c_{-1 / 2} f_{2}(\rho, \theta) \xi_{b}(z)\left|J_{z}=-\frac{1}{2}\right\rangle \\
c_{+1 / 2} f_{1}(\rho, \theta) \xi_{a b}(z)\left|J_{z}=+\frac{1}{2}\right\rangle \\
c_{-3 / 2} f_{3}(\rho, \theta) \xi_{a b}(z)\left|J_{z}=-\frac{3}{2}\right\rangle
\end{array}\right)
$$

and

$$
\left|F_{z}=3 / 2, \nu=\downarrow\right\rangle=\left(\begin{array}{l}
c_{+3 / 2} f_{0}(\rho, \theta) \xi_{a b}(z)\left|J_{z}=+\frac{3}{2}\right\rangle \\
c_{-1 / 2} f_{2}(\rho, \theta) \xi_{a b}(z)\left|J_{z}=-\frac{1}{2}\right\rangle \\
c_{+1 / 2} f_{1}(\rho, \theta) \xi_{b}(z)\left|J_{z}=+\frac{1}{2}\right\rangle \\
c_{-3 / 2} f_{3}(\rho, \theta) \xi_{b}(z)\left|J_{z}=-\frac{3}{2}\right\rangle
\end{array}\right) .
$$

Here $f_{m_{z}}(\rho, \theta)$ represents the in-plane part of the envelope function with envelope angular momentum $m_{z} \cdot \xi_{\nu_{z}}(z)$ is the vertical part of the envelope function, which can be bonding $\left(\nu_{z}=b\right)$ or antibonding $\left(\nu_{z}=a b\right) .^{22}\left|J_{z}\right\rangle$ represents the Bloch 


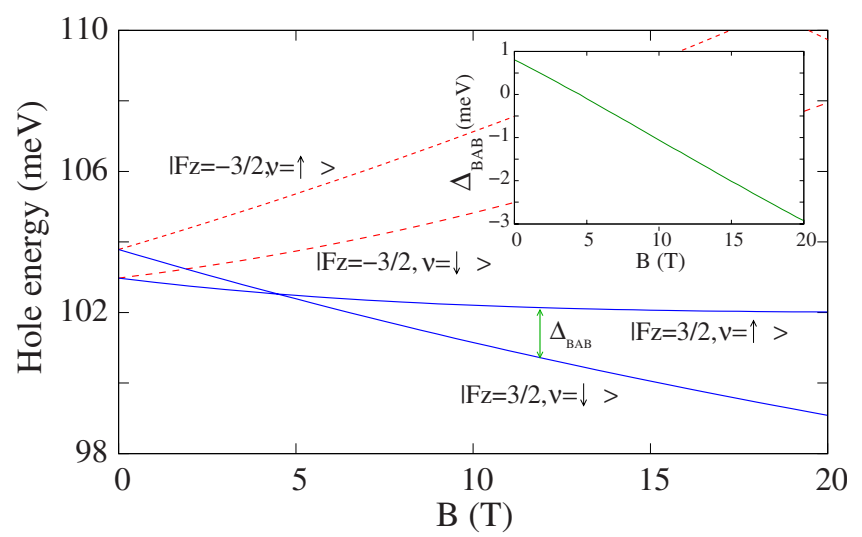

FIG. 1. (Color online) Hole energy levels in a CQD as a function of the magnetic field. The inset shows the splitting between the lowest "bonding" and "antibonding" levels, which is tuned by the field down to zero and then to negative values.

function with Bloch angular momentum $J_{z}$, and $c_{J_{z}}$ is a coefficient that we determine numerically. ${ }^{23}$ In the absence of magnetic fields, $\left|F_{z}=3 / 2, \nu=\uparrow\right\rangle$ is Kramers degenerate with $\left|F_{z}=-3 / 2, \nu=\downarrow\right\rangle$, and $\left|F_{z}=3 / 2, \nu=\downarrow\right\rangle$ with $\left|F_{z}=-3 / 2, \nu=\downarrow\right\rangle$. However, the field lifts this degeneracy favoring the states with positive $F_{z}$ (Ref. 21) so that Eqs. (1) and (2) soon describe the two lowest-lying levels. We shall focus on these states.

For a usual CQD, the first component of the spinors above is strongly dominant. Thus, $\left|F_{z}=3 / 2, \nu=\uparrow\right\rangle$ is essentially a bonding $\mathrm{HH}$ with $m_{z}=0$ and $\left|F_{z}=3 / 2, \nu=\downarrow\right\rangle$ is essentially an antibonding $\mathrm{HH}$ with $m_{z}=0$. However, the minor LH component with $m_{z}=1\left(J_{z}=+1 / 2\right)$ can bring about important changes in the molecular behavior. This is because its molecular character (bonding or antibonding) is opposite to that of the dominant component. In the spinor given by Eq. (1), the LH component is antibonding so that it unstabilizes the bonding $\mathrm{HH}$. By contrast, in the spinor of Eq. (2) it is bonding and therefore stabilizes the antibonding $\mathrm{HH}$. This results in an overall reduction in $\Delta_{\mathrm{BAB}}$, and we say that the tunnel coupling strength has been reduced by the SOinduced valence mixing. ${ }^{19}$ Since the tunneling of LHs is much larger than that of HHs, this effect can be important even if the weight of the LH component is small. Indeed, for long interdot distances, where the tunneling of HHs is negligible compared to that of LHs, this is responsible for the reversal of $\left|F_{z}=3 / 2, \nu=\uparrow\right\rangle$ and $\left|F_{z}=3 / 2, \nu=\downarrow\right\rangle$ spinors observed by Doty et al. ${ }^{20}$ The main message of this paper is that a similar control on $\Delta_{\mathrm{BAB}}$ can be achieved not by changing the interdot distance but simply by applying a longitudinal magnetic field. This is because the dominant HH component has $m_{z}=0$, but the relevant LH component has $m_{z}=1$. Thus, while the $\mathrm{HH}$ component is a little sensitive to the field, the LH component is stabilized; its relative weight increases gradually with $B$.

To illustrate this principle, in Fig. 1 we plot the lowest hole levels of a $\mathrm{GaAs} / \mathrm{Al}_{0.3} \mathrm{Ga}_{0.7} \mathrm{As} \mathrm{CQD}$ versus magnetic field. The two CQDs are identical and form a homonuclear QD molecule. ${ }^{24}$ Solid lines are used for states with positive $F_{z}$ and dashed ones for states with negative $F_{z}$. When the field is switched on, $\left|F_{z}=3 / 2, \nu=\uparrow\right\rangle$ becomes the ground state, but for stronger fields $(B>4.5 \mathrm{~T})$, it is replaced by $\left|F_{z}=3 / 2, \nu=\downarrow\right\rangle$. This is because $\left|F_{z}=3 / 2, \nu=\downarrow\right\rangle$ has a larger LH component than $\left|F_{z}=3 / 2, \nu=\uparrow\right\rangle$ (Ref. 19) and is then

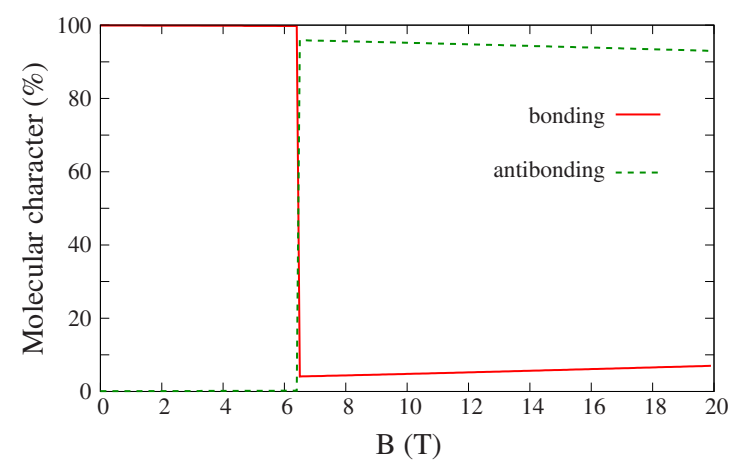

FIG. 2. (Color online) Molecular character of the ground state as a function of the magnetic field. The solid line indicates the weight of bonding character, while the dashed line indicates that of antibonding character. Note the abrupt transition, from mostly bonding to mostly antibonding, is at $B \sim 4.5 \mathrm{~T}$.

more strongly affected by the field. One can identify the energy splitting between these two states with $\Delta_{\mathrm{BAB}}$, which we plot in the inset (solid line). As can be seen, the magnetic field tunes $\Delta_{\mathrm{BAB}}$ from initially positive values down to zero and then to negative values. Actually, the negative values induced at large magnetic fields can be even larger (in magnitude) than those at zero field. This shows that the longitudinal magnetic field is a versatile tool to manipulate the tunnel coupling strength. We note that $\Delta_{\mathrm{BAB}}$ can be tuned to zero because $\left|F_{z}=3 / 2, \nu=\uparrow\right\rangle$ and $\left|F_{z}=3 / 2, \nu=\downarrow\right\rangle$ have different chirality symmetry, which is preserved by the field. If this was not the case, the two states would anticross and $\Delta_{\mathrm{BAB}}$ would never be zero.

For the magnetic-field-induced bonding-antibonding reversal to take place, a number of conditions must be met. First, the ground state at zero field must be $\left|F_{z}=3 / 2, \nu=\uparrow\right\rangle$, which is usually the case for small interdot distances. 19,20 Otherwise the ground state will always be $\left|F_{z}=3 / 2, \nu=\downarrow\right\rangle$ as its splitting with $B$ is larger. Second, the LH component must be sizable. This depends on the constituent materials and the strain fields. ${ }^{19}$ We tested if the ground state reversal of Fig. 1 is also feasible in InGaAs/GaAs CQDs and found that it takes place at much larger values of $B$ (not shown) because the biaxial strain severely weakens the HH-LH mixing. ${ }^{14}$

Since Luttinger spinors contain an admixture of bonding and antibonding components, it is worth quantifying to which extent the ground state reversal reported in Fig. 1 implies a change in the molecular character. To this end, in Fig. 2 we plot the weight of the ground state spinor components with bonding (solid line) and antibonding (dashed line) characters as a function of the magnetic field, as inferred from the squared coefficients of Eqs. (1) and (2). Up to $B$ $\sim 4.5 \mathrm{~T}$, when the ground state is $\left|F_{z}=3 / 2, \nu=\uparrow\right\rangle$, the molecular character is over $99 \%$ bonding. For stronger fields, with $\left|F_{z}=3 / 2, \nu=\downarrow\right\rangle$ as the ground state, it is $93 \%-96 \%$ antibonding. This clearly confirms that the ground state is switched from mostly bonding to mostly antibonding. The stronger admixture of $\left|F_{z}=3 / 2, \nu=\downarrow\right\rangle$ is connected with its larger LH component, as mentioned above.

So far we have investigated the simple case of a single hole in two identical QDs. A more realistic scenario, which could serve to test our predictions, is studied next. We consider two dots with slightly different size and charge with an interacting electron-hole pair (exciton), as in usual optically charged self-assembled CQDs. In this kind of system, the 


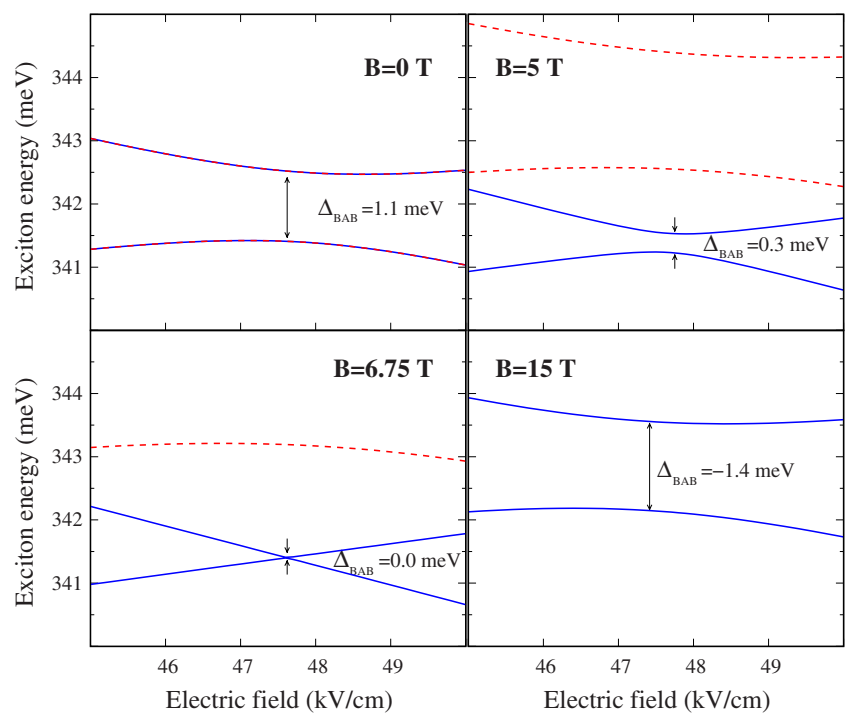

FIG. 3. (Color online) Exciton energy as a function of the electric field for an asymmetric CQD subject to different magnetic fields. The solid lines denote the states involving hole levels with $F_{z}=3 / 2$, while the dashed lines denote those involving $F_{z}=-3 / 2$. The gap between the bonding and antibonding exciton levels is tuned with the magnetic field.

particles are mostly localized in the bigger QD so that the molecule is strongly heteronuclear. However, one can apply an electric field along the molecular axis to induce a resonance of the atomiclike levels of either electrons or holes. ${ }^{12}$ In this way, a homonuclearlike behavior is restored for the chosen carrier. At the resonant value of the electric field, the emission spectrum of the exciton reveals an anticrossing between the bonding and antibonding states, whose magnitude is precisely $\Delta_{\mathrm{BAB}} \cdot{ }^{11,12,25}$

In Fig. 3 we plot the low-energy exciton states as a function of the magnetic field for an asymmetric CQD. ${ }^{26}$ The electron state is calculated using an effective mass approach and the electron-hole Coulomb interaction term is solved using a configuration interaction scheme with the Hartree products arising from the two lowest (bonding and antibonding) electron and hole states. ${ }^{19}$ An electric field is applied, which brings the hole into resonance while leaving the electron in the higher dot. The fine structure arising from electron-hole exchange interaction is neglected here as it is not relevant for the message. Four different cases are illustrated. At zero magnetic field (top left panel), an anticrossing of $\Delta_{\mathrm{BAB}}$ $=1.1 \mathrm{meV}$ is observed. At $B=5 \mathrm{~T}$ (top right panel), this gap is reduced to $\Delta_{\mathrm{BAB}}=0.3 \mathrm{meV}$, as the hole states $\mid F_{z}$ $=3 / 2, \nu=\uparrow\rangle$ and $\left|F_{z}=3 / 2, \nu=\downarrow\right\rangle$ are now closer together. At $B=6.75 \mathrm{~T}$ (bottom left panel), the gap collapses, indicating that the two hole levels are degenerate. Note that this occurs despite the fact that the different vertical confinements of the top and bottom dots break the chirality symmetry. The reason for this is that the resonant electric field restores an effective chirality for the molecular states. ${ }^{19,20}$ Finally, at $B=15 \mathrm{~T}$ (bottom right panel) $\left|F_{z}=3 / 2, \nu=\downarrow\right\rangle$ is by far the hole ground state, and the anticrossing gap $\left.\left(\Delta_{\mathrm{BAB}}=-1.4 \mathrm{meV}\right)\right)$ is even larger in magnitude than it was at zero field. Further, it has negative sign because the ground state is mostly antibonding.

In conclusion, we have demonstrated that the tunnel coupling of QD molecules containing resonant holes can be controlled using longitudinal magnetic fields. The tunnel coupling strength can be reduced down to zero and to large negative values, thus switching from a ground state with strong bonding character to one with strong antibonding character. This tuning of the molecular spectrum is exclusive of artificial molecules due to the SO-induced valence band mixing, and it can be exploited to produce quantitative or qualitative changes in the response of devices based on vertically CQDs.

We thank P. Hawrylak, M. Doty, and D. Gammon for critical reading of the manuscript. Support from the Ramon y Cajal Program, MEC Project No. CTQ2008-03344 and Cineca Calcolo Parallelo 2008 is acknowledged.

${ }^{1}$ G. W. Bryant, Phys. Rev. B 47, 1683 (1993).

${ }^{2}$ A. W. Holleitner, R. H. Blick, A. K. Hüttel, K. Eberl, and J. P. Kotthaus, Science 297, 70 (2002).

${ }^{3}$ M. Pi, A. Emperador, M. Barranco, F. Garcias, K. Muraki, S. Tarucha, and D. G. Austing, Phys. Rev. Lett. 87, 066801 (2001).

${ }^{4}$ M. Bayer, P. Hawrylak, K. Hinzer, S. Fafard, M. Korkusinski, Z. R. Wasilewski, O. Stern, and A. Forchel, Science 291, 451 (2001).

${ }^{5}$ S. A. Wolf, D. D. Awschalom, R. A. Buhrman, J. M. Daughton, S. von Molnár, M. L. Roukes, A. Y. Chtchelkanova, and D. M. Treger, Science 294, 1488 (2001).

${ }^{6}$ Nano-optoelectronics: Concepts, Physics and Devices, edited by M. Grundmann (Springer, Berlin, 2002).

${ }^{7}$ A. J. Nozik, Physica E (Amsterdam) 14, 115 (2002).

${ }^{8}$ F. Troiani, E. Molinari, and U. Hohenester, Phys. Rev. Lett. 90, 206802 (2003)

${ }^{9}$ E. A. Stinaff, M. Schneibner, A. S. Bracker, I. V. Ponomarev, V. L. Korenev, M. E. Ware, M. F. Doty, T. L. Reinecke, and D. Gammon, Science 311, 636 (2006).

${ }^{10}$ A. K. Hüttel, S. Ludwig, H. Lorenz, K. Eberl, and J. P. Kotthaus, Phys. Rev. B 72, 081310(R) (2005).

${ }^{11}$ H. J. Krenner, M. Sabathil, E. C. Clark, A. Kress, D. Schuh, M. Bichler, G. Abstreiter, and J. J. Finley, Phys. Rev. Lett. 94, 057402 (2005).

${ }^{12}$ A. S. Bracker, M. Schneiber, M. F. Doty, E. A. Stinaff, I. V. Ponomarev, J. C. Kim, L. J. Whitman, T. L. Reinecke, and D. Gammon, Appl. Phys. Lett. 89, 233110 (2006).

${ }^{13}$ G. Burkard, G. Seeling, and D. Loss, Phys. Rev. B 62, 2581 (2000).

${ }^{14}$ M. Korkusinski and P. Hawrylak, Phys. Rev. B 63, 195311 (2001).

${ }^{15}$ D. Bellucci, F. Troiani, G. Goldoni, and E. Molinari, Phys. Rev. B 70, 205332 (2004).

${ }^{16}$ D. Kim, S. E. Economou, S. C. Badescu, M. Scheibner, A. S. Bracker, M Bashkansky, T. L. Reinecke, and D. Gammon, arXiv:0809.1673v1 [Phys. Rev. Lett. (in press)].

${ }^{17}$ L. G. G. V. Dias da Silva, J. M. Villas-Boas, and S. Ulloa, Phys. Rev. B 76, 155306 (2007).

${ }^{18}$ B. D. Gerardot, D. Brunner, P. A. Dalgarno, P. Öhberg, S. Seidl, M. Kroner, K. Karrai, N. G. Stoltz, P. M. Petroff, and R. Warburton, Nature (London) 451, 441 (2008).

${ }^{19}$ J. I. Climente, M. Korkusinski, G. Goldoni, and P. Hawrylak, Phys. Rev. B 78, 115323 (2008).

${ }^{20}$ M. F. Doty, J. I. Climente, M. Korkusinski, M. Scheibner, A. S. Bracker, P. Hawrylak, and D. Gammon, arXiv:0804.3097v1.

${ }^{21}$ L. G. C. Rego, P. Hawrylak, J. A. Brum, and A. Wojs, Phys. Rev. B 55, 15694 (1997).

${ }^{22}$ When the two QDs of the molecule are identical, bonding and antibonding are simply the symmetric and antisymmetric linear combinations of atomic orbitals.

${ }^{23}$ For holes (electrons), $f_{m}(\rho, \theta)$ is expanded on a basis of 6 (3) Bessel functions and $\xi_{\nu_{z}}(z)$ on a basis of 30 harmonics. The coefficients are found with an exact diagonalization procedure.

${ }^{24}$ The QDs are $2.5 \mathrm{~nm}$ high and have a radius of $15 \mathrm{~nm}$. The interdot barrier is $3.5 \mathrm{~nm}$ long with height $V_{c}=200 \mathrm{meV}$. GaAs Luttinger parameters are used, $\gamma_{1}=6.98, \gamma_{2}=2.06$, and $\gamma_{3}=2.93$ (Ref. 27).

${ }^{25}$ G. Ortner, M. Bayer, Y. Lyanda-Geller, T. L. Reinecke, A. Kress, J. P. Reithmaier, and A. Forchel, Phys. Rev. Lett. 94, 157401 (2005).

${ }^{26}$ The upper (lower) QD is now $2.7(2.3) \mathrm{nm}$ high. The electron effective mass is $m_{e}^{*}=0.067 m_{0}$ ( $m_{0}$ is the free electron mass), the conduction barrier potential is $V_{c}^{e}=260 \mathrm{meV}$, and the dielectric constant is $\epsilon=12.4$. The rest of the parameters are as in Ref. 24.

${ }^{27}$ I. Vurgaftman, J. R. Meyer, and L. R. Ram-Mohan, J. Appl. Phys. 89, $5815(2001)$ 